\title{
Spontaneous Pneumomediastinum and Bilateral Pneumothoraces in a Patient with Bleomycin-Induced Pneumonitis
}

\author{
Matthieu Barras, Marc Uhlmann \\ Division of Internal Medicine, Regional Hospital Riviera-Chablais, Monthey, Switzerland
}

Received: 04/09/2017

Accepted: 23/09/2017

Published: 09/10/2017

How to cite this article: Barras M, Uhlmann M. Spontaneous pneumomediastinum and bilateral pneumothoraces in a patient with bleomycin-induced pneumonits. EJCRIM 2017;4: doi:10.12890/2017_000727

Conflicts of Interests: The Authors declare that there are no competing interests.

This article is licensed under a Commons Attribution Non-Commercial 4.0 License

\section{ABSTRACT}

Bleomycin lung toxicity is well established and can manifest as bleomycin-induced pneumonitis, but pneumomediastinum and pneumothorax are very rare complications. We report the case of a 73-year-old woman, recently treated with bleomycin for Hodgkin's disease, who was admitted for bleomycin-induced pneumonitis. Two weeks later she had a pneumomediastinum with extensive subcutaneous emphysema and small bilateral pneumothoraces. Three months after that she was readmitted for dyspnoea. The CT scan showed complete regression of the pneumomediastinum but extensive bilateral ground-glass infiltrates. The patient died from respiratory failure 2 weeks later.

\section{LEARNING POINTS}

- Respiratory investigation before initiation of bleomycin treatment and then close follow-up during treatment of any abnormalities found is mandatory, as bleomycin -induced toxicity can lead to fibrosis and secondary pneumothorax /pneumomediastinum with high morbidity/mortality.

- Bleomycin-induce pneumonitis (BIP) is managed with bleomycin discontinuation (Grade 1A) and system corticosteroid (Grade 1B).

- Supplemental oxygen is discouraged for BIP, but indicated for conservative management of pneumothoraces, so this case was managed with limited oxygen supplementation (aiming for oxygen saturation of 92-94\%).

\section{KEYWORDS}

Bleomycin-induced pneumonitis, pneumomediastinum, pneumothorax

\section{CASE DESCRIPTION}

A 73-year-old woman who had never smoked and had been recently diagnosed with Hodgkin's lymphoma stage IIIB, was treated with two cycles of ABVD (adriamycin, bleomycin, vinblastine and dacarbazine) with a total dose of bleomycin of $200 \times 10^{3} \mathrm{units}$ for $57 \mathrm{~kg}$. The PET-CT scan after two cycles showed complete radiological and metabolic remission. However, a few days before the third cycle of chemotherapy, the patient developed rapidly progressive dyspnoea and a dry cough limiting her physical activity, together with mild hypoxaemia and bilateral lung crackles. A CT scan of the chest showed airspace consolidation, linear opacities, septal thickening especially at the bases, and traction bronchiectasis (Fig. 1). We diagnosed bleomycin-induced pneumonitis (BIP) because of the temporal relationship between the lung fibrosis and the treatment, and because of the absence of a differential diagnosis. We therefore stopped bleomycin and started systemic 
corticosteroids ( $1 \mathrm{mg} / \mathrm{kg}$ of body weight). Two weeks later, the patient complained of facial and neck swelling, dyspnoea and rhinolalia. There was no history of emesis, trauma or strenuous physical activity. Oxygen saturation was $90 \%$ by pulse oximetry (SpO2) on room air. A chest CT scan showed, in addition to the above findings, a pneumomediastinum, small bilateral pneumothoraces and subcutaneous emphysema (Fig. 2). The patient was admitted for close observation and supplemental oxygen, aiming for $\mathrm{SpO}_{2}$ of $92-94 \%$. Cough and constipation were treated proactively to minimize subsequent Valsalva manoeuvres. A Gastrografin transit time x-ray, bronchoscopy and gastroscopy were performed but did not reveal the source of the mediastinal air leak. Bronchoalveolar lavage showed a mixed alveolitis with mild eosinophilia and ruled out pneumocystis pneumonia. The patient was discharged home after 1 month in hospital. A follow-up CT scan 2 months after the initial admission showed subtotal resolution of the pneumomediastinum. One month later, the patient was readmitted for dyspnoea. The CT scan showed complete regression of the pneumomediastinum but extensive bilateral ground-glass infiltrates. The patient's respiratory condition rapidly worsened and she died from respiratory failure 2 weeks later. Retrospective review of a pre-ABVD CT scan showed discrete pre-existing subpleural lung infiltrates. possiblv caused bv lvmphoma or pre-existing interstitial lung disease.

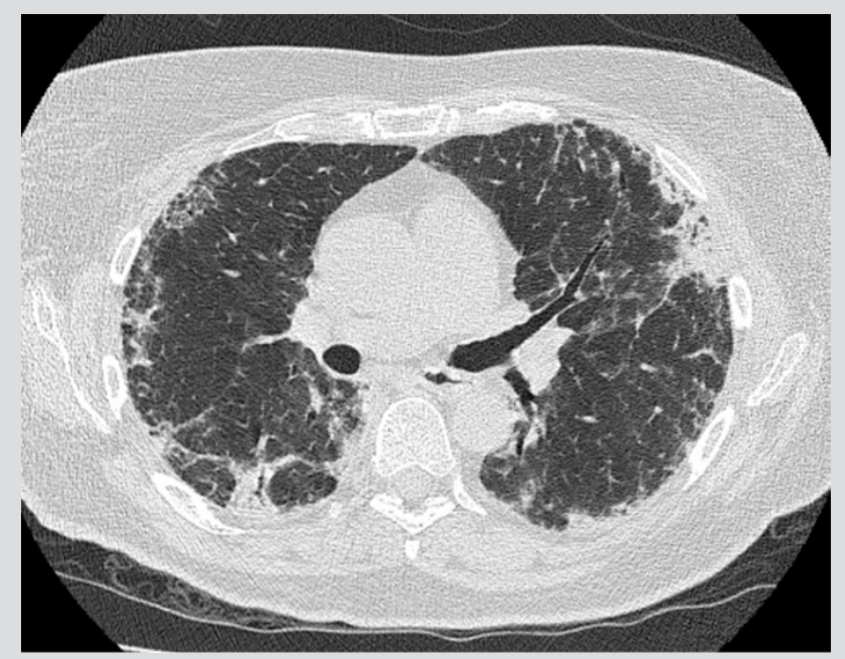

Figure 1. Diagnosis of bleomycin-induced pneumonitis: airspace consolidation, linear opacities, septal thickening and traction bronchiectasis

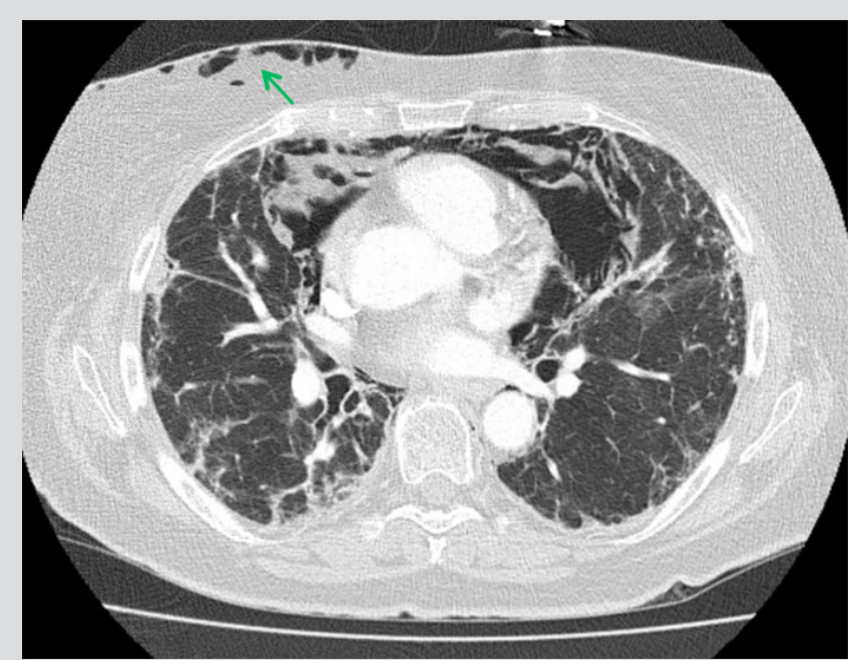

Figure 2. Pneumomediastinum with subcutaneous emphysema (green arrow)

Methods and procedures

We searched PubMed using the keywords 'bleomycin','pulmonary interstitial disease,',pneumomediastinum','pneumothorax' and 'treatment'. Our search retrieved 10 articles, all of which were case reports published from 1985 to 2017, and described 10 adult male patients and 1 male child. Four of the patients had pneumomediastinum and pneumothorax, five had pneumomediastinum only and two pneumothorax only. Nine were treated with steroids and five with antibiotics. Three patients completely recovered in 1-7 months, seven died after a few days of respiratory distress, and two died from septic shock after 7 and 30 days ${ }^{[1-10]}$.

\section{DISCUSSION}

After the diagnosis was made, we stopped bleomycin treatment and started systemic corticosteroid therapy (1 mg/kg of body weight). The cytotoxic agent bleomycin can sometimes induce potentially fatal pulmonary toxicity, also known as BIP. BIP starts with an inflammatory reaction modifying the lung architecture and leading to pulmonary fibrosis. Besides bleomycin discontinuation, there is no effective treatment for BIP in humans, although systemic corticosteroids are widely prescribed ${ }^{[11]}$. We treated our patient with limited oxygen supplementation, aiming for $\mathrm{SpO}_{2}$ of $92-94 \%$. Data obtained from animal studies suggest oxygen supplementation should not be given during bleomycin therapy as high oxygen concentrations encourage free radical production, which may increase bleomycin lung toxicity ${ }^{[12-13]}$.

We prescribed codeine and laxatives to avoid spontaneous cough and constipation (Valsalva manoeuvres). Spontaneous pneumomediastinum (SPM) is defined as radiologically detected free air in the mediastinum, without preceding trauma ${ }^{[14]}$. It is mostly seen after intrathoracic pressure shifts leading to alveolar rupture and dissection ${ }^{[15]}$. Pneumomediastinum in patients with pulmonary fibrosis is thought to be the 
result of rupture of alveoli or honeycomb cysts ${ }^{[12,15]}$. Sahni et al. published a review that included 201 patients with SPM ${ }^{[12]}$.

The most frequent triggering events were emesis, strenuous physical activity and cough. SPM is usually managed conservatively (analgesics, rest, oxygen therapy, bronchodilators and occasionally antibiotic treatment) and follows a benign course ${ }^{[12]}$. Our patient did not receive antibiotics as she never showed any symptoms or signs of infection.

A retrospective review of a pre-ABVD CT scan showed pre-existing interstitial lung disease (discrete subpleural lung infiltrates) and moderately reduced CO diffusion. The pre-ABVD spirometry was normal. The origin of the interstitial lung disease has not been determined, and may have affected lung pathology.

Retrospectively, based on the pre-treatment abnormalities, thecase could have been handled differently. There is noabsolutecontraindication but perhaps the bleomycin dosage should have been discussed.

This case report raises various points. First, before bleomycin treatment is started, respiratory investigations are mandatory. If any abnormality is detected, closer follow-up of the patient during treatment must be established. Second, supplemental oxygen is discouraged for BIP but indicated for conservative management of pneumothoraces, making oxygen management difficult in this situation. Third, despite numerous investigations (including CT scans, a Gastrografin transit time x-ray, gastroscopy and bronchoalveolar lavage), we never found a source of mediastinal air leak, which highlights the difficulty of managing these BIP-related complications.

\section{REFERENCES}

1. Frusch N, Servais S, De Prijck B, Corhay JL, Beguin Y, Louis R, et al. Spontaneous pneumomediastinum caused by bleomycin-induced pneumonitis. Acta Clin Belg 2012;67:370371.

2. Araujo MS, Fernandes FL, Kay FU, Carvalho CR. Pneumomediastinum, subcutaneous emphysema, and pneumothorax after a pulmonary function testing in a patient with bleomycin-induced interstitial pneumonitis. J Bras Pneumol 2013;39:613-619.

3. Sikdar T, MacVicar D, Husband JE. Pneumomediastinum complicating bleomycin related lung damage. Br J Radiol 1998;71:1202-1204.

4. Jennane S, Haidouri S, Zine Filali K, Ahchouch S, Mahtat EM, Doghmi K, et al. Fatal bleomycin induced pneumomediastinum and bilateral pneumothorax. Rev Pneumol Clin 2017;73:153-156.

5. Namitha R, Nimisha KP, Yusuf N, Rauf CP. Air leak: an unusual manifestation of organizing pneumonia secondary to bleomycin. Lung India 2017;34:173-175.

6. Keijzer A, Kuenen B. Fatal pulmonary toxicity in testis cancer with bleomycin-containing chemotherapy. J Clin Oncol 2007;25:3543-3544.

7. Jain TP, Thulkar S, Saha S, Bakhshi S, Dominic J. Extensive pneumothorax, pneumomediastinum and surgical emphysema as a complication of bleomycin therapy. Pediatr Radiol 2005;35:1227-1229.

8. Briassoulis G, Hatzis T, Paphitis C, Stinios J. Acute spontaneous pneumomediastinum in a child with Hodgkin's disease and pulmonary fibrosis. Pediatr Hematol Oncol 1999;16:175-180.

9. Doll DC. Fatal pneumothorax associated with bleomycin-induced pulmonary fibrosis. Cancer Chemother Pharmacol 1986;17:294-295.

10. Leeser JE, Carr D. Fatal pneumothorax following bleomycin and other cytotoxic drugs. Cancer Treat Rep 1985;69:344-345.

11. Sleijfer S. Bleomycin-induced pneumonitis. Chest 2001;120:617-624.

12. Sahni S, Verma S, Grullon J, Esquire A, Patel P, Talwar A. Spontaneous pneumomediastinum: time for consensus. N Am J Med Sci 2013;5:460-464.

13. Grahmann PR, Brauer M, Hüter L, Sayer H, Neumann R, Braun RK. Respiratory failure and pulmonary fibrosis as a late side-effect after chemotherapy-induced by oxygen administration. Pneumologie 2005:59:763-769.

14. Svedbrand C, Lange P, Nielsen K. Spontaneous mediastinal emphysema. Ugeskr Laeger 2016;178:V08150653.

15. Caceres M, Ali SZ, Braud R, Weiman D, Garrett HE Jr. Spontaneous pneumomediastinum: a comparative study and review of the literature. Ann Thorac Surg 2008;86:962-966.

16. Fujiwara T. Pneumomediastinum in pulmonary fibrosis. Detection by computed tomography. Chest 1993;104:44-46. 